\title{
Canadian Drug Regulatory Framework
}

\author{
L. Kelly, M. Lazzaro, C. Petersen
}

\begin{abstract}
The role of regulatory drug submission evaluators in Canada is to critically assess both the data submitted and the sponsor's interpretation of the data in order to reach an evidence-, and context- based recommendation as to the potential benefits and potential harms (i.e., risks) associated with taking the drug under the proposed conditions of use. The purpose of this document is to outline the regulatory framework in which this assessment occurs, including: defining what "authorization to market a drug in Canada" means, in terms of the role of the sponsor, the responsibility of Health Canada in applying the Food and Drugs Act prior to and after marketing authorization, and the distinction between regulatory authorization versus physician authorization; highlighting organizational, process and legal factors within Health Canada related to authorization of clinical trials and authorization to market a drug; considerations during the review process, such as regulatory and scientific issues related to the drug, patient populations and trial designs; application of international guidelines, and decisions from other jurisdictions; regulatory realities regarding drug authorization, including the requirement for wording in the Product Monograph to accurately reflect the information currently available on the safe and effective use of a drug, and that hypothesis-confirming studies are essential to regulatory endorsement; current issues related to the review of therapies for dementia, such as assessing preventative treatments, and therapies that have symptomatic versus disease-modifying effects, statistical issues regarding missing data, and trial design issues.
\end{abstract}

RÉSUMÉ: Cadre de réglementation canadien sur les médicaments. Au Canada, le rôle de ceux qui évaluent les soumissions à l'organisme de réglementation est critique, tant en ce qui concerne l'évaluation des données soumises que leur interprétation par le promoteur afin d'arriver à une recommandation basée sur les données et sur le contexte quant aux bénéfices et aux dangers (c.-à-d. aux risques) potentiels associés à la prise du médicament, tels que décrits dans la soumission. Le but de ce document est de faire une esquisse du cadre réglementaire régissant cette évaluation, soit : la définition de ce que signifie «l'autorisation de commercialiser un médicament au Canada », en termes du rôle du promoteur, de la responsabilité de Santé Canada dans l'application de la législation sur les denrées alimentaires et les médicaments avant et après la commercialisation et la distinction entre l'autorisation réglementaire et l'autorisation du médecin; les facteurs organisationnels, procéduraux et légaux propres à Santé Canada concernant l'autorisation d'essais cliniques et l'autorisation de commercialiser un médicament; les éléments considérés au cours du processus de révision tels les questions réglementaires et scientifiques concernant le médicament, les populations de patients et les plans d'études; l'application des lignes directrices internationales et les décisions d'autres juridictions; les dispositions réglementaires concernant l'autorisation de commercialisation, dont les exigences concernant la façon d'énoncer l'information disponible sur l'utilisation sûre et efficace d'un médicament dans la monographie du produit et la nécessité d'études confirmant l'hypothèse pour l'approbation réglementaire; les problèmes actuels concernant la révision de traitements pour la démence comme l'évaluation de traitements préventifs et de médicaments qui ont des effets symptomatiques versus des effets sur l'évolution de la maladie, les problèmes statistiques concernant les données manquantes et les problèmes concernant les plans d'étude.

Can. J. Neurol. Sci. 2007; 34: Suppl. 1 - S3-10

\section{INTRODUCTION}

- An application to Health Canada (HC) to market a new drug in Canada is done by the sponsoring pharmaceutical company; the application typically includes animal data, chemistry and manufacturing data, and the results from clinical trials of various phases. The amount of data submitted in support of a new chemical entity frequently reaches hundreds of volumes.

- The role of drug evaluators is to critically assess both the data and the sponsor's interpretation of the data in order to determine, on a population basis, the potential benefits and the potential harms (i.e., risks) associated with taking the drug. Evaluators apply regulatory expertise to reach an evidence- and contextbased recommendation as to the risk-benefit profile of the drug within the context of the proposed conditions of use.

\section{Purpose}

- The purpose of this document is to outline the regulatory framework in which Canadian review of drug applications occurs, including: the mandate dictated by Canada's Food and Drug Act/Food and Drug Regulations (CFDA/F\&DR), and the

From CNSD, BCANS, Therapeutic Products Directorate, Tunney's Pasture, Ottawa, ON, Canada.

Received OCtOBER 7, 2005. ACCEPTED IN FINAL FORM November 22, 2005. Reprint requests to: Lisa Kelly, Finance Building, CNSD, BCANS, Therapeutic Products Directorate, Tunney's Pasture, Address locator: 0202A1, Ottawa, Ontario, K1A 1B9, Canada. 
significance of specific wording in the Act; process factors such as the organizational review streams, and legal issues such as "proprietary information"; international guidelines provided by the International Conference on Harmonization (ICH), EMEA and the FDA; various examples of primary general considerations during review, and several issues more specific to review of drugs for Alzheimers disease (AD).

\section{Authorization by Health Canada to Market in Canada: WHAT IT MEANS}

\section{Legal Framework of the Food and Drugs Act}

- The review of data submitted in support of the marketing of a new drug, or a new claim for a marketed drug, occurs within the legal framework of the CFDA/F\&DR Part C, Drugs, Division 8; (see Health Canada website: http://laws.justice.gc.ca/en/showdoc/cr/C.R.C.-c.870/bo-ga:1 Cgb:s C 08 001//en\#anchorbo-gal C-gb:s C 08001

\section{Under C.08.001:}

- "New Drug" means a drug that contains or consists of a substance that has not been sold in Canada for sufficient time and in sufficient quantity to establish in Canada the safety and effectiveness of that substance for use as a drug.

- Thus, the definition of "new drug" applies not only to drugs not marketed in Canada, but also applies to those drugs that are not yet "sold in Canada for sufficient time and in sufficient quantity....". The term "new drug" applies to both prescription and over the counter drug products, including new formulations of marketed drugs as well as combinations of already-marketed drugs.

- Before any "new drug" can legally be sold in Canada, its sponsor must obtain authorization from Health Canada via the submission of data and proposed labeling to Health Canada. The submission is to be examined for evidence of manufacturing quality, and safety and efficacy in relation to its recommended purpose and conditions of use i.e., for a specific condition, patient population, dosing schedule, contraindications, warnings etc.

\section{Under C.08.002:}

(1) No person shall sell or advertise a new drug unless:

a) the manufacturer of the new drug has filed with the Minister a new drug submission relating to the new drug that is satisfactory to the Minister.

b) the Minister has issued, pursuant to section C.08.004, a notice of compliance to the manufacturer of the new drug in respect of the new drug submission.

c) the notice of compliance in respect of the submission has not been suspended.

(2)A new drug submission shall contain sufficient information and material to enable the Minister to assess the safety and effectiveness of the new drug, including the following...."

Then follows a complete list of all the necessary contents of a new drug submission, including: "a draft of every label";

"detailed reports of tests made to establish the safety of the new drug for the purpose and under the conditions of use recommended"; "substantial evidence of the clinical effectiveness of the new drug for the purpose and under the conditions of use recommended".
For those drug submissions which are considered by Health Canada to be satisfactory a Notice of Compliance (NOC) is issued to the sponsor.

\section{Regulatory Recognition of InHerent Limitations to Drug SAFETY}

- It is inherent in regulatory authorization that claims as to efficacy or safety are limited to those conditions of use described in the labeling; that is, the patient population, the age, the dosage and duration, etc.

- In Canada, the Act/F\&DR specifically further recognizes that, even within the parameter of specified conditions of use, what can be known about a drug from pre-market trials, both in terms of safety and efficacy, is only a small proportion of what may become apparent after a number of years of market exposure. The term "Notice of Compliance" (NOC) is used to signify that the submission for the new drug "is satisfactory to the Minister" in terms of "sufficient information and material to enable the Minister to assess the safety and effectiveness of the new drug". As well, because years of actual clinical use are required before the safety profile of a drug can be considered to be established, a newly-authorized drug continues to be termed a "new drug" by Health Canada for some variable period subsequent to authorization. The point at which a new drug can be considered to have been "sold for sufficient time and sufficient quantity in Canada" such that it is no longer termed a "new" drug is currently under discussion at Health Canada.

- For those drugs considered as a "new drug", a subsequent submission by the sponsor requesting, for example a new claim, is therefore termed a "Supplement to a New Drug Submission". Thus, the complete wording in this section of the Act/F\&DR allows for recognition that a change to the claims for a drug decreases our comfort level, even if there have been years of prior exposure under the original claim.

- Health Canada's role doesn't end when drug products are authorized for sale, as post-market surveillance is essential to maintaining the balance between health benefits and risks. Marketed Health Products Directorate works collaboratively with other Directorates to perform post-marketing surveillance activities, including monitoring of adverse drug reaction reports, evaluating product effectiveness, and recommending appropriate regulatory action as needed, based on re-evaluation of riskbenefit profile.

\section{Authorization CANNOT OCCUR IN THE ABSENCE OF AN APPLICATION BY THE SPONSOR}

- The decision to submit a drug application for review is made by the sponsor. A variety of business factors may affect a sponsor's decision whether and when to submit in Canada, including profitability and Canadian patent laws. Thus, while absence of a drug from the Canadian market could mean that Health Canada rejected the application, it could also mean that the application was never submitted to Canada. Health Canada has no authority to compel a sponsor to apply for authorization of a therapeutic product, nor to determine when an application will be submitted. - In the absence of a submission from a sponsor, Health Canada cannot authorize a therapeutic product, regardless of whether the product is authorized elsewhere. The mandate that regulatory 
agencies have, that is to render evidence- and context- based decisions regarding therapeutic products, requires access to the actual data, and this access can only occur by means of an application from the manufacturer.

- Just as the sponsor has the responsibility to submit a complete dataset in order to request authorization to market their product in Canada, so has Health Canada the reciprocal responsibility to perform a rigorous assessment of the benefits and risks of the therapeutic product, and render an evidence-, and context-, based decision on these data. This is a federally-mandated responsibility which occurs in response to an application from the sponsor, regardless of whether the drug has been authorized or not, for any conditions of use, in another country.

\section{Health Canada's Responsibility to Scrutinize Sponsor's INTERPRETATION}

- This responsibility makes good sense from a scientific point of view because, as with scientific journal peer review, a primary aspect of therapeutic product regulatory review is scrutinizing the sponsor's interpretation of the data for strengths and weaknesses. Clinical trials are scientific and medical experiments, and therefore, as with all scientific endeavours, the findings are rarely absolute but rather are subject to interpretation. Scrutiny is required of interpretations to ensure they have an objective and rigorous basis. Given this, and the impact on patients of therapeutic product authorization or rejection, examination of the sponsor's interpretation by more than one organization is important peer review, despite the fact that major regulatory agencies around the world share guidelines (see later section for more information on international standards).

- In addition, the mandate of Health Canada to render an evidence-based decision also allows the Canadian regulatory and health care context to be taken into consideration. Some factors depend on the country or region in which the decision is being made, including the availability of alternative therapies, clinical practices, the health care system, and on the level of risktolerance/risk-aversion inherent to that society.

Authorization does not Mean a DRUg CANNot haRm AN INDIVIDUAL PATIENT (nor that every patient will experience benefit)

- Both physicians and patients need to be aware that all drug products have both desirable and undesirable potential effects. Thus, all drug products, including those products authorized by regulatory agencies, carry the "possibility of harm" (i.e., risk) to any individual patient. This "double-edged sword" is inherent with the use of drugs, and is well summarized in an excerpt from the excellent document on the science of the safety of medicines, co-authored by the Uppsala Monitoring Centre, and the WHO Collaborating Centre for International Drug Monitoring, and entitled Viewpoint Part 1: Watching for Safer Medicines (http://www.who-umc.org/graphics/6996.pdf) From page 5 of the document: "The truth about the nature of drugs has long been understood by scientists, but the message has not reached the general public. In its report for 1969-70, the UK Committee on the Safety of Drug included the following: 'No drug which is pharmacologically effective is entirely without hazard. The hazard may be insignificant or may be acceptable in relation to the drug's therapeutic action. Furthermore, not all hazards can be known before a drug is marketed; neither tests in animals nor clinical trials will always reveal all the possible side effects of a drug. These may only be known when the drug has been administered to large numbers of patients over considerable periods of time' ".

- Thus, the issue is twofold: not only is it known that all drugs have undesirable side effects, but also it is recognized that testing cannot reveal all of these side effects. For example, at the premarket stage, it is typically not tenable to conduct clinical trials which are large enough to detect rare adverse events or trials that are long enough in duration to detect adverse events which may appear only after chronic use.

- There is also a third factor underlying the concept that authorization does not mean that a drug cannot harm an individual patient. Both the benefits and harms of a drug are referred to as "potential" because while they are documented within clinical trials to occur in specific percentages of all the observed patients using the product, they may or may not occur for any specific individual patient. That is, any individual patient taking the drug may or may not experience benefit, and may or may not experience a specific side effect. Thus, the use of the terms potential benefits and potential harms is in part reflective of the inherent "population viewpoint" of regulatory assessments.

- It is of note that Canadian regulations do not strictly require that a drug demonstrate a unique benefit over other drugs in the same class in order to receive authorization; rather, as for all new drug submissions, "substantial evidence of the clinical effectiveness of the new drug for the purpose and under the conditions of use recommended" is required. The issue of whether a drug demonstrates a unique benefit over other drugs in the class may, however, become relevant when weighing the adverse event profile of a drug against its benefits, particularly if the drug exhibits a unique safety concern.

- In summary, authorization by Health Canada -i.e. the issuance of a NOC - does not mean that a drug can do no harm, nor does it mean that every individual patient will experience benefit. Rather, "satisfactory to the Minister" is interpreted by Health Canada to mean that, on a population basis, the potential harms (i.e., risks) are judged to be acceptable given the specific conditions of use, considering the potential benefits and the alternatives available at the time of NOC issuance.

\section{Regulatory Authorization versus Physician AUTHORIZATION}

- The distinction between patient populations versus individual patients is important to the effective management of the risks of prescription drugs, as the distinction is reflected in the two levels of "authorization": 1) by the regulatory agency, for the population and 2) by the physician, for the individual patient. Thus, regulatory agencies, health care providers and patients each represent three complementary arenas of risk/benefit assessments: by regulatory agencies; by prescribers; and by patients.

- Regulatory agencies authorize or reject drug applications for a specific patient population(s) or condition(s), based on an evidence-based judgement call as to whether the "potential for harm" (ie risks) of the product are acceptable in light of: 
-its potential benefits for a specific patient population(s)

-conclusions about the manageability of the potential harms, either through the PM and/or well-designed risk management programs, and

-risk/benefit profile of alternative therapies available in Canada.

Regulatory agencies ensure that for those drugs which are authorized for the specific condition of use there is accurate, balanced and substantial information provided in the Product momograph (PM).

- Physicians evaluate the risks and benefits in terms of each specific patient, providing a professional opinion as to whether the authorized drug is right for that individual. This professional opinion for individual patients includes any uses for which the drug is not specifically indicated by the regulatory agency i.e., "off-label" use.

- Just as the PM represents the population risk-benefit assessments by regulatory, so do clinical practice guidelines represent risk-benefit assessments within that therapeutic area by the medical community. While the two distinct domains necessarily overlap, the information in clinical practice guidelines is inherently broader in scope than that in a PM, and often there is information that is not present in the PM, such as recommendations regarding additional conditions of use, and comparative safety or efficacy data. It may be that these data do not meet the regulatory requirements that would support inclusion of that information in the PM, or were not submitted to the regulatory agency. Thus, both the PM and clinical practice guidelines provide physicians with informationthat is overlapping but not identical, for making evidence-based decisions for treating patients. In parallel, the art and science of drug review (i.e. population risk-benefit judgement based on critical analysis and interpretation of experimental data) overlaps with, but is demonstrably different from, the art and science of clinical practice (i.e. risk-benefit judgement for individual patients based on diagnosis, and treatment recommendations). Regulatory science and clinical practice inform and complement each other, but are also very separate spheres of knowledge.

- Finally, the patient evaluates the risks and benefits of the prescription drug in terms of their own personal values, as they are the ones who ultimately experience the outcomes. The labeling for each drug includes a section for the consumer, which includes a lay language description of the conditions of use and warnings.

- These three distinct but related levels are each critical to optimal use of a prescription drug, and none should be working in isolation from the others.

- For those drugs that are unavailable for sale in Canada, Health Canada considers that clinical trials, including open-label, are the safest possible mechanism through which patients can have access. In exceptional circumstances, i.e., when clinical trials are inappropriate or when a patient is not eligible or able to enroll into a trial, access to such drugs may be available through Health Canada's Special Access Programme to patients with serious or life-threatening conditions, on a compassionate or emergency basis, when conventional therapies have failed, are unsuitable or are unavailable. (see HC website:

http://www.hc-sc.gc.ca/dhp-mps/acces/drugs-drogues/index_e.html).

\section{Health Canada: Salient Organizational Information}

\section{Process Issues}

- The submission of data by the sponsor starts the formal review process that will end up with either a positive or negative decision by Health Canada. The review process is targeted to be completed within a set length of time (i.e., 300 days for a New Drug Submission), with documentation to be prepared by Health Canada evaluators concerning the interpretations and conclusions that led to the recommendation. When questions or interpretation issues or safety concerns arise during the review, Health Canada evaluators can ask the sponsor for clarifications, and for such additional information as re-analysis of the data, or rationales for specific sponsor conclusions. Once a submission is accepted for review, sponsors may be permitted to submit new efficacy data only if responding to a formal negative disposition from Health Canada. In contrast, sponsors are required to submit all safety data as they become available, as this may impact the outcome of the review.

- In general, the therapeutic area of an application determines where it is reviewed. In the case of submissions related to Alzheimers Disease, the applications are reviewed by the Central Nervous System Division (CNSD), located within the Bureau of Cardiology, Allergy and Neurological Sciences (BCANS). BCANS is one of various Bureaux within the Therapeutic Products Directorate (TPD) responsible for submission evaluation. Therapeutic Products Directorate is in turn located within the Health Products and Food Branch (HPFB). For overview of Directorate and Branch responsibilities, see the respective websites:

http://www.hc-sc.gc.ca/hpfbdgpsa/aboutus_e.html http://www.hc-sc.gc.ca/ hpfb-dgpsa/tpddpt/ aboutus_e.html and http://www.hc-sc.gc.ca/ hpfbdgpsa/ aboutus_e.html.

\section{Clinical Trial Authorization versus Drug Authorization}

- All clinical trials proposed to be conducted within Canada, for either non-authorized drug products, or for a non-authorized condition of use for an authorized drug product, must be assessed and authorized by Health Canada prior to initiation of the trial. This assessment and authorization is performed within TPD, by the Clinical Trials unit within the Senior Medical Advisor Bureau. This bureau is separate from those bureaux reviewing submissions for drug authorization.

- The clinical trial application requests permission to distribute the drug to responsible clinical investigators that are named in the application. Health Canada must review the application and notify the sponsor within 30 days if the application is found to be deficient.

- The Food and Drugs Act and Regulations provide authority to Health Canada to regulate the sale of drugs for the purposes of use in human clinical trials. Part C, Division 5 of the Regulations defines specific Clinical Trial Application, and Clinical Trial Application Amendment, requirements for the sale and importation of drugs for use in human clinical trials in Canada. The Regulations are consistent with the principles, definitions and standards found in the Health Canada / ICH Guidance Documents E6: Good Clinical Practice: Consolidated Guideline, E8: General Considerations for Clinical Trials and E2A: Clinical Safety Data Management: Definitions and 
Standards for Expedited Reporting. These guidance documents, developed through the ICH process have been adopted by Health Canada. Together, they define parameters for the design, conduct, performance, monitoring, auditing, recording, analysis and reporting of clinical trials. For more information, see website: http://www.hc-sc.gc.ca/dhp-mps/prodpharma/ applicdemande/guide-ld/clini/index_e.html.

Notice of Compliance with Conditions (NOC/c) for treatment of serious, life-threatening or severely debilitating conditions

- An NOC/c is authorization to market a drug i.e., a Notice of Compliance (NOC), with the condition that the sponsor undertake additional studies to verify the clinical benefit. The NOC, qualifying under the NOC/c policy, is issued under section C.08.004 or C.08.005 of the Food and Drug Regulations. Verbatim from the web fact sheet on NOC/c.

- The purpose of the $\mathrm{NOC} / \mathrm{c}$ policy is to a) provide patients suffering from serious, life-threatening or severely debilitating diseases or conditions with earlier access to promising new drugs and b) create a mechanism to ensure that a manufacturer that has a drug authorized under this policy conducts confirmatory studies to verify the clinical benefit of the drug and further establish the safety profile.

- Authorization under the NOC/c policy may be granted for a drug product with promising clinical benefit for a serious, lifethreatening or severely debilitating condition, providing the drug possesses an acceptable safety profile based on a benefit/risk assessment, and is found to be of high quality. Prior to authorization, the sponsor must undertake in writing to design, carry out in a timely fashion, and report on well-designed confirmatory studies to verify the clinical benefit of the drug. For the purposes of monitoring the safety of the drug product, sponsors must agree to enhanced post-market surveillance and reporting requirements. For the purposes of assuring the safe use of the drug product, the conditions under which the drug product is approved must be clearly reflected and highlighted in the labeling. The sponsor may also be requested to undertake to comply with restrictions imposed by Health Canada on the advertisement and/or distribution of the drug.

- An NOC/c was issued to Lundbeck Canada Inc for Ebixa (memantine hydrochloride) in 2004. For more details see the Health Canada website:

http://www.hc-sc.gc.ca/dhp-mps/prodpharma/noticesavis/conditions/index_e.html.

For additional information on the $\mathrm{NOC} / \mathrm{c}$ policy, refer to Health Canada website:

http://www.hc-sc.gc.ca/dhp-mps/prodpharma/applicdemande/guide-ld/compli-conform/noccg_accd_2006_e.html.

\section{Proprietary Information}

- Health Canada has a legal obligation to maintain the confidentiality of therapeutic product application-related information; Canadian courts have indicated a general duty of confidentiality in a number of cases relating to the disclosure of therapeutic product application information. Therefore, information submitted to Health Canada by the sponsor regarding a drug product is considered to be the "proprietary information" of that sponsor, regardless of whether the drug is approved or not. This generally includes information concerning whether or not an application has actually been filed, as well as certain confidential material contained in any application filed with Health Canada.

- The release of confidential therapeutic product application information typically occurs either through the Access to Information Act (form available at: http://www.tbssct.gc.ca/tbsf-fsct/350-57_e.asp ) or under the protection of a Confidentiality Order. The Canadian office of the manufacturer of a therapeutic product should be contacted directly to request such proprietary information regarding therapeutic product development or the regulatory status of their product. Note that Health Canada normally is not able to comment on public statements made by the sponsor regarding a drug which is not authorized.

- When necessary for public safety, otherwise confidential information may be released by Health Canada.

- The Summary Basis of Decision Initiative is a joint project between TPD and Biologics and Genetics Therapeutic Directorate, with the goal of developing and implementing a process to publish documents for public consumption that summarize and explain product-specific regulatory decisions. The documents will include regulatory, safety, efficacy and quality considerations. For more information see:

http://www.hc-sc.gc.ca/dhp-mps/prodpharma/sbd-smd/index e.html - For further information on the process aspects of drug review in Health Canada see:

http://www.hc-sc.gc.ca/ahc-asc/pubs/hpfb-dgpsa/accesstherapeutic acces-therapeutique e.html

\section{Considerations in a Health Canada Regulatory Review}

The in-depth evaluation of the potential harms (undesirable effects) and potential benefits (desirable effects) of a therapeutic product must always be done in the context of the proposed conditions of use by the sponsor. The following are examples of some of the contextual factors that must be addressed by therapeutic product evaluators:

\section{International Guidelines}

- There is considerable formal international discussion on standards in evidence-requirement and data interpretation in drug authorization, within the forum of the "International Conference on harmonisation of Technical Requirements for Registration of Pharmaceuticals for Human Use" (ICH; for further information see:

http://www.ich.org/cache/compo/276-254-1.html).

The US, the 'European Union and Japan are the three major players, with additional jurisdictions such as Canada participating through "observer" status.

- Health Canada is committed to consultation on, and implementation of, ICH guidelines in a timely, transparent and expeditious manner. To date, all finalized $\mathrm{ICH}$ documents have been formally adopted by Health Canada (for further information, see Health Canada website at:

http://www.hc-sc/gc.ca/dhp-mps/prodpharma/applicdemande/guide-ld/ich/index e.html

Scroll down and click on ICH ). Thus, these guidelines represent our baseline standards - although we retain the right to add Canadian statements - and can all be accessed on the ICH website. Supplementary internal guidances may be developed by 
Health Canada for a specific situation, as for example, with combination products.

- An additional important source of regulatory standards are the guidelines from the European Agency for the Evaluation of Medicinal Products (EMEA; see

http://www.emea.europa.eu/htms/human/humanguidelines/back ground.htm) as well as those of the FDA.

(http://www.fda.gov/cder/guidance/index/htm)

- These various international guidelines reflect international thinking on both general regulatory principles, and within specific therapeutic areas. For example, regarding clinical data, the issues these documents address include: minimum exposure requirements, confounds in trial designs, statistical principles; views on diagnostic and outcome measures in established and emerging therapeutic areas.

\section{Examples of General Factors:}

\section{Related to the Drug}

- Is it the first of a new class of drugs, or does it belong to an established class? If the latter, how does it compare to others in the class in terms of unique safety concerns, or a unique benefit? - Is the drug intended as monotherapy or as adjunctive therapy? - Does the drug provide symptomatic relief i.e., anti-emetics, analgesics, and most CNS-active drugs, or is it curative i.e., antibiotics.

-What is the post marketing experience from other countries?

- Are there emerging risks associated with the drug as seen from current literature/expert sources?

- Have there been any regulatory actions in other countries?

\section{Related to the patient population/disease}

- Are the patients children, adults or elderly? Mostly female or mostly male?

- Are the patients generally healthy, with the desired benefit of drug use to prevent the onset of a disease e.g. Alzheimer's disease, or are they very sick, with the desired benefit of drug use to be life-saving e.g., a cure for AIDS.

- Is the drug intended for acute, intermittent or chronic use?

- Are there other available drugs for the disease? What is the comparative profile for safety and efficacy?

- Prevalence of the condition, and morbidity and mortality consequent to it.

- Patient acceptance of the risks/adverse reaction profile.

- Practice of medicine and societal demands.

\section{Related to Trial Design}

- Did each clinical trial use a validated tool or scale to measure the effect of the therapeutic product? Are the patients in the trial representative of those who will be treated with the product if authorized? Have the patients been diagnosed according to current accepted criteria for the condition being studied? Do the trials meet the internationally accepted standards outlined in $\mathrm{ICH}$ guidelines? Does the trial design allow meaningful interpretation of the data, or are there confounding factors, statistical or clinical, for example, that interfere with interpretation of the findings? Are there positive results from more than one Phase III efficacy trial? How many efficacy trials were negative and how many were failed (i.e., comparator also failed to show superiority to placebo?). Is the side-effect profile consistent across all studies?

- Given that the number of patients needed to provide a useful picture of the side effect profile is generally far greater than that needed to show that the drug works, was the number of patients in the trials sufficient from a safety perspective? How many and how long should extension trials be? Are there active comparators?

\section{Consultations}

- Expert consultation both internal and external occurs during Health Canada reviews, and Scientific Advisory Panels may also be consulted (see Health Canada website:

http://hc-sc.gc.ca/dhp-mps/prodpharma/activit/sci-consult/index e.html)

Health Canada is currently developing principles and documents for the Good Review Practices arm of the HPFB Therapeutic Access Strategy (see website:

http://www.hc-sc.gc.ca/dhpps/prodpharma/activit/proj/practicepratique/index e.html).

\section{Factoring in Decisions of other Jurisdictions}

- The same reasons that support the careful scrutiny of the sponsors interpretation of the data also support the judicious use of the thinking and assessments of other agencies (see page 3 in this document, "Responsibility to Scrutinize Sponsor's Interpretation").

- Thus, if decisions about the product have been made by other major regulatory agencies, they are factored in during review by Health Canada. When available, review reports by foreign agencies are also considered, in order to optimize both the effectiveness and the speed of Canadian reviews. The considerable international discussion on standards in drug data are recognized by Canada in our formal adoption of $\mathrm{ICH}$ guidelines, as discussed above.

- It is also important to note that, in addition to consideration of foreign decisions and review reports regarding a therapeutic product, Canadian reviews utilize adverse events reports from other countries where the product is marketed. Such data must always be assessed in terms of the likelihood that a problem, if there was one, could in fact be detected; this likelihood depends on the country from which the data originate, the length of time the product has been on the market, the numbers of patients exposed to the product, the conditions of use, and the frequency with which various side effects are reported. For example, was the patient population one for which it may be less likely that side effects would be noticed and reported i.e., elderly, debilitated patients versus young, otherwise healthy patients versus very sick patients?

- Many factors influence the number of reports received, and in most situations there is considerable under-reporting of adverse reactions with both voluntary and mandatory spontaneous surveillance systems.

\section{Summary}

$\infty$ This is only a brief sketch of some of the considerations that are part of the assessment when a drug application is reviewed by Health Canada. 
- International guidelines represent only general principles and criteria, and therefore cannot reflect the range of possible individual data characteristics of every submission. Meeting the guidelines thus cannot be considered a "guarantee" of authorization, as the findings for any specific submission may be insufficient evidence to support authorization. The presence of a signal in the data of a potential issue, whether safety- or efficacyrelated, requires additional scrutiny and regulatory assessment.

$\rightarrow$ The type of information assessed ranges from an interpretation by the sponsor of the entire submission package, through all relevant contextual factors, down to the details of individual case reports. Thus it can be seen that drug evaluation calls for the ability to be able to move from a birds eye view of the forest, to zoom down into the tiniest detail on the bark of an individual tree, and then move back out again for the contextual big picture impact of the detail.

\section{Some Regulatory Realities Regarding Drug Authorization \\ Distinction between "Proposed" and "Authorized" Wording for Labeling}

- Along with the sponsor's study reports, interpretations and conclusions, the submission also contains the sponsor's proposed Product Monograph (PM) and other labeling documents. The sponsor's proposed wording for this document is a distilled representation of precisely how they interpret the totality of their data in terms of actual patient use, including all claims, dosing and safety issues; the document therefore represents a pivotal tool for the evaluator in assessing the sponsor's interpretation. It is rare that regulatory interpretation of the data coincides exactly with that of the sponsor.

- For those products for which authorization to be sold in Canada is granted, the sponsor and Health Canada jointly develop a final version of the PM that accurately reflects the information currently available on the safe and effective use of the product, and provides the information required by each of clinicians and patients for optimal use of the therapeutic product. These "conditions of use" include for example, which patients can benefit, who should not be exposed to the product, how the product should be used, what are the potential harms. Thus, the assessment by a specific regulatory authority of a drug submission is reflected not only in whether or not the drug was granted authorization, but also in the precise conditions of use for which it was authorized, as reflected in the labeling.

- Product Monograph wording is also critical because it determines precisely what can be claimed in advertising (see Health Canada website:

http://www.hc-sc.gc.ca/dhp-mps/advert-publicit/index_e.html).

See also website of the Pharmaceutical Advisory Advertising Board: http:// www.paab.ca).

All information in the PM, including that found only in cited references, can be used for promotional purposes, with criteria dependent on the context.

\section{AVAILABLE EVIDENCE DELINEATES OUTCOME OF REVIEW}

- Regulatory agencies can authorize and label drugs only on the basis of available evidence of benefits and risks. Thus, while "hypothesis generating" studies are the undisputed foundation of all research advances, and are typically rich in information which catalyzes further studies, it is the narrow category of "hypothesis confirming" studies which is essential to the population-based decisions of regulatory drug authorization.

- These study designs should include such aspects as: a welldefined patient population for which validated diagnostic tools exist; clinically-relevant, validated, reliable and state-of-the-art measuring scales for that population; and adequate control groups. As noted by the ICH and EMEA documents, noninferiority trials require a pre-defined treatment difference ("delta"), which should be of a clinically meaningful size, and also tend to require a more rigorous adherence to protocol than do superiority trials in order to ensure interpretability. This is in part because, whereas in superiority trials the sponsor's desired outcome (i.e. detection of a treatment difference) inherently provides a validation of the sensitivity of the trial design for detection of the difference, in the case of the non-inferiority trial the desired outcome could be a reflection of non-rigorous trial conduct, or poor efficacy in both treatment arms, or an inappropriately large delta.

- The number of patients exposed through both controlled and open-label trials must be considered to provide an adequate safety database for authorization. The size of the safety database required for any individual submission depends on a variety of factors, including the therapeutic area involved, the signals seen in the data, and the extent of previous marketing exposure of the drug in Canada and in other major jurisdictions.

- Trials designs must reflect clinical practice issues and statistical soundness such that the data support an appropriate INDICATION. For example, if the expectation is that a drug will be used as adjunctive treatment, the clinical trials must be designed to provide interpretable data in support of such use, including appropriate stratification and powering if both monotherapy and adjunctive therapy arms are included in the trial.

- In addition to the importance of available evidence, there is also a role in regulatory assessments for any precedents set by decisions made on recent submissions in the same therapeutic area, both in terms of authorization and PM wording. As outlined in this document, drug evaluation decisions reflect various aspects of reviewing, including national societal values, availability of alternative therapies on the Canadian market and precedents set by previous labels.

\section{ReVIEW ISSUES WITH TherapIES FOR DEMENTIA}

- In general, for each review issue, current international guidelines are applied, with a framework for a consultative process if controversies emerge, that provides a means to obtain input from various external experts in Canada in order to achieve an evidence-based resolution.

- Current therapies for dementia are considered to provide symptomatic treatment only, rather than modifying the rate of disease progression. Several methods for detecting and providing evidence of progression-modifying effects are currently being discussed internationally, including randomized start or withdrawal study designs, and biochemical and brain-imaging markers.

- For preventative treatment in $\mathrm{AD}$, generally three potential target periods are acknowledged: primary prevention occurs 
during the latent, non-symptomatic stage to prevent disease onset; secondary prevention during the possible "prodromal stage" to prevent reaching the full syndrome; tertiary prevention after disease onset, to prevent or slow disease progression. The acceptable level of risk associated with the use of a drug varies across these periods, with the lowest risk tolerance for populations containing individuals who may never experience the disease.

- Regarding current symptomatic treatments: It has been proposed that $\mathrm{AD}$ patients showing symptomatic improvement on a drug may not in fact be returning to their previous state, but rather achieving a new state which may not be adequately captured with current measuring scales. Clearly, the more accurately measurement tools can reflect the clinical impact of the treatments, the more sound the regulatory process.

- As noted by both ICH and EMEA guidelines, it is important to adopt a conservative approach to missing data, but it is of note that there is no universally accepted methodological approach for handling missing data. Although "Last Observation Carried Forward" is a widely used method, it is recognized that it may not be the most conservative in clinical situations where the patient's condition is expected to deteriorate over time, as in the case of $\mathrm{AD}$ patients. Pre-specification of the primary efficacy dataset, with justification, is essential, as is comparison of the results from that dataset to results from other efficacy datasets. - In line with international standards, a minimum of two positive, well-designed and controlled trials demonstrating efficacy and safety are typically required to support an NOC for an indication in $\mathrm{AD}$, any $\mathrm{AD}$-variant or non-Alzheimer's type dementia. Negative and failed trials are also evaluated, and weighed into the assessment. In all cases, trials designs should include clinically meaningful diagnostic criteria and validated efficacy outcome measures appropriate for the assessment of change in clinically relevant symptoms of the disease. Note that authorization under the NOC/c policy may be supported with only one such trial, given the requirement for confirmatory data. The potential risk : benefit ratio of a drug is likely to be viewed differently by regulatory in the case of drugs with an established safety /efficacy profile, as compared to those lacking substantial post-marketing experience ie new chemical entities.

- Current "standard of care" for AD in North America dictates that all patients diagnosed with mild to moderate AD are treated with cholinesterase inhibitors. Therefore, it is now considered unethical to include a placebo-only arm in $\mathrm{AD}$ trials, such that trials must have either "placebo add-on" arms, active comparator arms, or include only patients who cannot tolerate cholinesterase inhibitors. This change to trial design may lead to a need for more sensitive, yet specific, outcome measures, including potential functional composite measures addressing clinical dimensions not emphasized in current scales. Regulatory principles regarding composite endpoints can be found in international guideline documents, including text which is not specific to any therapeutic area such as the EMEA document "Points to Consider on Multiplicity Issues in Clinical Trials" (see EMEA website: http://www.emea.europa.eu/pdfs/human/ewp/090899en.pdf)

and the ICH document "E9: Statistical Principles for Clinical Trials" (see ICH website:

http://www.ich.org/ LOB/media/MEDIA485.pdf).

\section{Legal Disclaimer}

This explanatory document represents the current thinking of the Central Nervous System Division of the Therapeutic Products Directorate on this topic. It does not create or confer any rights for or on any person and does not operate to bind Health Canada or the public. 